\title{
A Mathematical Approach to Establishing Constitutive Models for Geomaterials
}

\author{
Guang-hua Yang, ${ }^{1}$ Yu-xin Jie, ${ }^{2}$ and Guang-xin $\mathrm{Li}^{2}$ \\ ${ }^{1}$ Guangdong Provincial Research Institute of Water Conservancy and Hydropower, \\ Guangzhou 510610, China \\ ${ }^{2}$ State Key Laboratory of Hydroscience and Engineering, Tsinghua University, \\ Beijing 100084, China
}

Correspondence should be addressed to Yu-xin Jie; jieyx@tsinghua.edu.cn

Received 1 March 2013; Accepted 25 May 2013

Academic Editor: Pengcheng Fu

Copyright (C) 2013 Guang-hua Yang et al. This is an open access article distributed under the Creative Commons Attribution License, which permits unrestricted use, distribution, and reproduction in any medium, provided the original work is properly cited.

\begin{abstract}
The mathematical foundation of the traditional elastoplastic constitutive theory for geomaterials is presented from the mathematical point of view, that is, the expression of stress-strain relationship in principal stress/strain space being transformed to the expression in six-dimensional space. A new framework is then established according to the mathematical theory of vectors and tensors, which is applicable to establishing elastoplastic models both in strain space and in stress space. Traditional constitutive theories can be considered as its special cases. The framework also enables modification of traditional constitutive models.
\end{abstract}

\section{Introduction}

The mechanical properties of geomaterials are complex and essential to make a numerical prediction; thus, many researchers have paid attention to constitutive relations of geomaterials. The simplest constitutive model for geomaterials is the elastic model, among which the common nonlinear models are the Cauchy elastic model, the hyperelastic model, and the hypoelastic model. The Cauchy elastic model assumes that the stress (or strain) in the material depends on the current strain (or stress) only, and not on its history. The constitutive equation for the hyperelastic model is established by the strain energy function or complement energy function. The hypoelastic model assumes that the stress state of an elastic material is associated with both the strain state and the stress path. The typical hypoelastic models are the E- $\mu$ and E$B$ models proposed by Duncan et al. $[1,2]$ and the K-G model [3-5].

According to the experimental results, most deformations of geomaterials are plastic deformations. Therefore, traditional plasticity theory has often been used to establish constitutive models for soil. For example, Drucker et al. [6] described the deformation property of soil by traditional elastoplastic theory and proposed a model with conical yield surface affected by hydrostatic pressure. Roscoe et al. [7] proposed a plastic cap model for normally consolidated clay, which is well known as the Cambridge model. Subsequently, Roscoe and Burland [8] modified the dilatancy equation in the Cambridge model and proposed a modified Cambridge model with elliptical yield surface. Wroth and Bassett [9] and Poorooshasb et al. [10] extended the model to sandy soil, and Yao et al. [11, 12] extended the model to sandy soil and overconsolidated soil. There are plenty of elastoplastic models, such as models with a single yield surface proposed by Desai et al. $[13,14]$ and Lade et al. [15-18], models with a double yield surface, and three surface models [19]. The concepts of the bounding surface [20-23] and the subloading surface [24, 25], endochronic theory [26], and disturbed states [27] have also been applied to establishing constitutive models for geomaterials.

In this paper, a theoretical framework on establishing constitutive models for geomaterials is proposed, the initial thought of which is provided by the first author in 1988 and in 1990s [28-31], and it has been implemented by some researchers to simulate the behavior of jointed rock masses [32] and soil-structure interface [33]. 


\section{Classical Elastoplastic Theory of Geomaterials}

The incremental form of a stress-strain relationship in traditional geomechanics is generally expressed as

$$
\mathrm{d} \sigma_{i j}=D_{i j k l} \mathrm{~d} \varepsilon_{k l} \text {. }
$$

Determining $D_{i j k l}$ is the major topic for constitutive models of geomaterials. Obviously, $D_{i j k l}$ can be obtained by fitting experimental data, given that experiments on stress and strain tensors are conducted. However, it is extremely difficult to do so. Therefore, experiments on the stress-strain relationship of geomaterials are usually conducted in principal stress/strain space; that is, only the relationship between the principal stress $\sigma_{i}(i=1,2,3)$ and the principal strain $\varepsilon_{i}(i=1,2,3)$, is obtained. To obtain $D_{i j k l}$, the constitutive tensor in general coordinate space should be derived from the stress-strain relationship in principal stress/strain space. From a mathematical point of view, these can be treated as the problems of coordinate transformation [34-36].

The relationship between the plastic strain increment and stress increment in principal stress/strain space is defined as

$$
\begin{gathered}
\left\{\mathrm{d} \varepsilon_{i}^{p}\right\}_{3 \times 1}=[A]_{3 \times 3}\left\{\mathrm{~d} \sigma_{i}\right\}_{3 \times 1}, \\
{[A]_{3 \times 3}=\left[\begin{array}{lll}
a_{11} & a_{12} & a_{13} \\
a_{21} & a_{22} & a_{23} \\
a_{31} & a_{32} & a_{33}
\end{array}\right],}
\end{gathered}
$$

where $a_{i j}$ are the functions of total stress $\sigma_{i}$, total strain $\varepsilon_{i}(i=$ $1,2,3)$ or stress path.

When the matrix rank of $[A]$ is 1 , or $|A|=0$, there exists a vector $\left\{\begin{array}{lll}\alpha_{1} & \alpha_{2} & \alpha_{3}\end{array}\right\}^{T}$ and coefficients $\beta_{1}, \beta_{2}, \beta_{3}$ to express [A] as

$$
[A]=\left\{\begin{array}{lll}
\beta_{1} & \beta_{2} & \beta_{3}
\end{array}\right\}^{T}\left\{\begin{array}{lll}
\alpha_{1} & \alpha_{2} & \alpha_{3}
\end{array}\right\},
$$

Therefore, substituting ( 3 ) into (2a) and ( $2 \mathrm{~b})$ gives

$$
\left\{\mathrm{d} \varepsilon_{i}^{p}\right\}=\left\{\begin{array}{lll}
\beta_{1} & \beta_{2} & \beta_{3}
\end{array}\right\}^{T}\left\{\begin{array}{lll}
\alpha_{1} & \alpha_{2} & \alpha_{3}
\end{array}\right\}\left\{\begin{array}{l}
\mathrm{d} \sigma_{i}
\end{array}\right\}
$$

that is,

$$
\left\{\mathrm{d} \varepsilon_{i}^{p}\right\}=\mathrm{d} \lambda\left\{\begin{array}{lll}
\beta_{1} & \beta_{2} & \beta_{3}
\end{array}\right\}^{T}
$$

where

$$
\mathrm{d} \lambda=\sum_{i=1}^{3} \alpha_{i} \mathrm{~d} \sigma_{i}
$$

According to (4b),

$$
\mathrm{d} \varepsilon_{1}^{p}: \mathrm{d} \varepsilon_{2}^{p}: \mathrm{d} \varepsilon_{3}^{p}=\beta_{1}: \beta_{2}: \beta_{3} .
$$

$\beta_{i}(i=1,2,3)$ is a function of $\sigma_{i}$ or $\varepsilon_{i}$. When $\boldsymbol{\beta}=\left\{\begin{array}{lll}\beta_{1} & \beta_{2} & \beta_{3}\end{array}\right\}^{T}$ is of a field with potential, there is a potential function $Q$ such that

$$
\beta_{i}=\frac{\partial Q}{\partial \sigma_{i}} .
$$

Substituting (7) into (4b), we have

$$
\mathrm{d} \varepsilon_{i}^{p}=\mathrm{d} \lambda \frac{\partial Q}{\partial \sigma_{i}} .
$$

If we assume that $\mathrm{d} \varepsilon_{i}^{p}$ and $\sigma_{i}$ have the same principal directions, the coordinate transformation can be expressed as follows:

$$
\mathrm{d} \varepsilon_{i j}^{p}=\mathrm{d} \varepsilon_{i}^{p} \frac{\partial \sigma_{i}}{\partial \sigma_{i j}} .
$$

Substituting (9) into (8) gives

$$
\mathrm{d} \varepsilon_{i j}^{p}=\mathrm{d} \lambda \frac{\partial Q}{\partial \sigma_{i j}} .
$$

Similarly, from elastic potentials theory, there is a potential function $W$ in principal stress space, and $\varepsilon_{i}$ is defined as

$$
\varepsilon_{i}=\frac{\partial W}{\partial \sigma_{i}} .
$$

If we assume that $\sigma_{i}$ and $\varepsilon_{i}$ have the same principal direction, the coordinate transformation can be expressed as follows:

$$
\varepsilon_{i j}=\varepsilon_{i} \frac{\partial \sigma_{i}}{\partial \sigma_{i j}} .
$$

Substituting (11) into (12),

$$
\varepsilon_{i j}=\frac{\partial W}{\partial \sigma_{i j}} .
$$

In conclusion, traditional plastic potential theory corresponds to the case that the matrix $[A]$ in $(2 a)$ and (2b) has rank 1, and $\beta$ can be expressed as the gradient vector of a potential function. Based on mathematical principles, a more general potential function-based constitutive framework can be established according to vector field theory and tensor theory as described below.

\section{Derivation of Constitutive Framework from Vector Field Theory}

Obviously, when the three principal components of the plastic strain increment, $\mathrm{d} \varepsilon_{i}^{p}(i=1,2,3)$, are considered to be components of a vector $\mathbf{d} \boldsymbol{\varepsilon}^{\mathbf{p}}$, the principal components can be expressed as three linearly independent $3 \mathrm{D}$ vectors by a vector fitting method. The gradient vectors of three linearly independent potential functions are selected as the linearly independent vectors.

When $\mathbf{d} \boldsymbol{\varepsilon}^{\mathbf{p}}$ is expressed in principal stress space, the coordinate orientations of $\mathbf{d} \boldsymbol{\varepsilon}^{\mathbf{p}}$ and $\sigma_{i}$ are the same, and $\Phi_{1}, \Phi_{2}, \Phi_{3}$ are three linearly independent potential functions in principal stress space, and then the following expression is obtained:

$$
\mathrm{d} \varepsilon_{i}^{p}=\sum_{k=1}^{3} \lambda_{k} \frac{\partial \Phi_{k}}{\partial \sigma_{i}},
$$


where $\lambda_{k}(i=1,2,3)$ are the coefficients. Suppose that the principal directions of $\mathbf{d} \boldsymbol{\varepsilon}^{\mathbf{p}}$ and $\sigma_{i}$ are the same, and we substitute (14) into (9), giving the tensor expression in general coordinate space as

$$
\mathrm{d} \varepsilon_{i j}^{p}=\sum_{k=1}^{3} \lambda_{k} \frac{\partial \Phi_{k}}{\partial \sigma_{i j}}
$$

Similarly, $\mathbf{d} \boldsymbol{\varepsilon}^{\mathbf{p}}$ can also be expressed in strain space. Let $\Psi_{1}, \Psi_{2}, \Psi_{3}$ be three linearly independent potential functions in principal strain space, and the following expression is obtained:

$$
\mathrm{d} \varepsilon_{i j}^{p}=\sum_{k=1}^{3} \mu_{k} \frac{\partial \Psi_{k}}{\partial \varepsilon_{i j}}
$$

Define the plastic stress increment as

$$
\left\{\mathrm{d} \sigma^{p}\right\}=\left[D_{e}\right]\left\{\mathrm{d} \varepsilon^{p}\right\},
$$

where $\left[D_{e}\right]$ is the elastic stiffness matrix, and then the expressions in stress space and strain space are

$$
\begin{array}{r}
\mathrm{d} \sigma_{i j}^{p}=\sum_{k=1}^{3} \beta_{k} \frac{\partial G_{k}}{\partial \sigma_{i j}}, \\
\mathrm{~d} \sigma_{i j}^{p}=\sum_{k=1}^{3} \alpha_{k} \frac{\partial F_{k}}{\partial \varepsilon_{i j}} .
\end{array}
$$

For the total stress and the total strain, consider the three principal stresses and the three principal strains as vectors in three-dimensional space with the same principal directions, and the following expressions are obtained:

$$
\begin{gathered}
\sigma_{i j}=\sum_{k=1}^{3} \eta_{k} \frac{\partial W_{k}}{\partial \varepsilon_{i j}}, \\
\varepsilon_{i j}=\sum_{k=1}^{3} \chi_{k} \frac{\partial \Omega_{k}}{\partial \sigma_{i j}},
\end{gathered}
$$

where $W_{k}, \Omega_{k}(i=1,2,3)$ are potential functions with linearly independent gradient vectors in strain space and stress space, respectively.

\section{Derivation of Constitutive Framework from Tensor Theory}

If $A_{i j}$ and $E_{i j}$ are symmetric second-order tensors with the same principal directions, the following equations are obtained according to tensor theory and vector fitting:

$$
\begin{aligned}
& A_{i j}=\sum_{k=1}^{3} \bar{\lambda}_{k} \frac{\partial I_{E k}}{\partial E_{i j}}, \\
& E_{i j}=\sum_{k=1}^{3} \bar{\beta}_{k} \frac{\partial I_{A k}}{\partial A_{i j}},
\end{aligned}
$$

where $I_{E k}(k=1,2,3)$ are three independent invariants of $E_{i j}$, and $I_{A k}(k=1,2,3)$ are three independent invariants of $A_{i j}$. For example, for the stress tensor $\sigma_{i j}$, the three independent invariants can be $I_{\sigma 1}=\sigma_{i i}, I_{\sigma 2}=(1 / 2) \sigma_{i j} \sigma_{j i}, I_{\sigma 3}=$ $(1 / 3) \sigma_{i k} \sigma_{k n} \sigma_{n m} ; I_{\sigma 1}=p, I_{\sigma 2}=q, I_{\sigma 3}=\theta$; or $I_{\sigma 1}=\sigma_{1}, I_{\sigma 2}=\sigma_{2}$, $I_{\sigma 3}=\sigma_{3}$, where $p$ is the mean stress, $q$ is the deviatoric stress, $\theta$ is the Lode's angle, and $\sigma_{1}, \sigma_{2}$, and $\sigma_{3}$ are the three principal stresses.

If $A_{i j}=\mathrm{d} \varepsilon_{i j}^{p}, E_{i j}=\sigma_{i j}, I_{\sigma k}(k=1,2,3)$ are invariants of $\sigma_{i j}$, it can be obtained from (21) that

$$
\mathrm{d} \varepsilon_{i j}^{p}=\sum_{k=1}^{3} \bar{\lambda}_{k} \frac{\partial I_{\sigma k}}{\partial \sigma_{i j}},
$$

which is equivalent to (15) when $\Phi_{k}=I_{\sigma k}$. then

$$
\text { If } A_{i j}=\mathrm{d} \varepsilon_{i j}^{p}, E_{i j}=\varepsilon_{i j}, I_{\varepsilon k}(k=1,2,3) \text { are invariants of } \varepsilon_{i j} \text {, }
$$

$$
\mathrm{d} \varepsilon_{i j}^{p}=\sum_{k=1}^{3} \bar{\alpha}_{k} \frac{\partial I_{\varepsilon k}}{\partial \varepsilon_{i j}} .
$$

If $A_{i j}=\varepsilon_{i j}, E_{i j}=\sigma_{i j}$, then

$$
\begin{aligned}
\sigma_{i j} & =\sum_{k=1}^{3} \bar{\beta}_{k} \frac{\partial I_{\varepsilon k}}{\partial \varepsilon_{i j}}, \\
\varepsilon_{i j} & =\sum_{k=1}^{3} \bar{\lambda}_{k} \frac{\partial I_{\sigma k}}{\partial \sigma_{i j}} .
\end{aligned}
$$

Equation (25) corresponds to (20), respectively, when $W_{k}=$ $I_{\varepsilon k}, \Omega_{k}=I_{\sigma k}$.

Similarly, if $A_{i j}=\Delta \varepsilon_{i j}, E_{i j}=\Delta \sigma_{i j}$, then according to (21), we have

$$
\Delta \varepsilon_{i j}=\sum_{k=1}^{3} \bar{\lambda}_{k} \frac{\partial I_{\sigma k}}{\partial\left(\Delta \sigma_{i j}\right)} .
$$

It should be noted that the derivations from vector field theory and from tensor theory are actually coincident. The potential functions in the derivation from vector field theory are functions of invariants, which degenerate to tensor form after composite derivation.

\section{Elastoplastic Matrix in Stress Space}

Without considering the effect of the Lode's angle $\theta$ and rotation of principal stress, the relations for the plastic strain increment and stress increment are expressed as

$$
\begin{aligned}
\mathrm{d} \varepsilon_{v}^{p} & =A \mathrm{~d} p+B \mathrm{~d} q, \\
\mathrm{~d} \bar{\varepsilon}^{p} & =C \mathrm{~d} p+D \mathrm{~d} q,
\end{aligned}
$$

where $A, B, C, D$ are parameters.

The following equation is obtained from the potential functions $\Phi_{1}=p, \Phi_{2}=q$ in (15) without considering the effect of the Lode's angle:

$$
\mathrm{d} \varepsilon_{i j}^{p}=\lambda_{1} \frac{\partial p}{\partial \sigma_{i j}}+\lambda_{2} \frac{\partial q}{\partial \sigma_{i j}} .
$$


In matrix form, this is

$$
\left\{\mathrm{d} \varepsilon^{p}\right\}=\lambda_{1}\left\{\frac{\partial p}{\partial \sigma}\right\}+\lambda_{2}\left\{\frac{\partial q}{\partial \sigma}\right\} .
$$

It is obvious that

$$
\lambda_{1}=\mathrm{d} \varepsilon_{v}^{p}, \quad \lambda_{2}=\mathrm{d} \bar{\varepsilon}^{p} .
$$

According to (27a), (27b), (29), and (30),

$$
\left\{\mathrm{d} \varepsilon^{p}\right\}=(A \mathrm{~d} p+B \mathrm{~d} q)\left\{\frac{\partial p}{\partial \sigma}\right\}+(C \mathrm{~d} p+D \mathrm{~d} q)\left\{\frac{\partial q}{\partial \sigma}\right\} .
$$

Since

$$
\mathrm{d} p=\left\{\frac{\partial p}{\partial \sigma}\right\}^{T}\{\mathrm{~d} \sigma\}, \quad \mathrm{d} q=\left\{\frac{\partial q}{\partial \sigma}\right\}^{T}\{\mathrm{~d} \sigma\}
$$

it follows that

$$
\begin{gathered}
\left\{\mathrm{d} \varepsilon^{p}\right\}=\left[C_{p}^{\sigma}\right]\{\mathrm{d} \sigma\} \\
{\left[C_{p}^{\sigma}\right]=A\left\{\frac{\partial p}{\partial \sigma}\right\}\left\{\frac{\partial p}{\partial \sigma}\right\}^{T}+B\left\{\frac{\partial p}{\partial \sigma}\right\}\left\{\frac{\partial q}{\partial \sigma}\right\}^{T}} \\
+C\left\{\frac{\partial q}{\partial \sigma}\right\}\left\{\frac{\partial p}{\partial \sigma}\right\}^{T}+D\left\{\frac{\partial q}{\partial \sigma}\right\}\left\{\frac{\partial q}{\partial \sigma}\right\}^{T},
\end{gathered}
$$

where $\left[C_{p}^{\sigma}\right]$ is the plastic compliance matrix.

Since $\{\mathrm{d} \varepsilon\}=\left\{\mathrm{d} \varepsilon^{e}\right\}+\left\{\mathrm{d} \varepsilon^{p}\right\}$, then

$$
\{\mathrm{d} \varepsilon\}=\left[C_{e p}^{\sigma}\right]\{\mathrm{d} \sigma\},
$$

where

$$
\left[C_{e p}^{\sigma}\right]=\left[C_{e}\right]+\left[C_{p}^{\sigma}\right]
$$

$\left[C_{e}\right]$ is the elastic compliance matrix and $\left[C_{e p}^{\sigma}\right]$ is the elastoplastic compliance matrix.

Therefore, the elastoplastic model in stress space can be established once the parameters $A, B, C$, and $D$ are determined. Note that $A, B, C$, and $D$ are not constants, which evolve with stress and strain, as presented in the following sections.

\section{Elastoplastic Matrix in Strain Space}

According to (17), the plastic stress increment is defined as $\left\{\mathrm{d} \sigma^{p}\right\}=\left[D_{e}\right]\left\{\mathrm{d} \varepsilon^{p}\right\}$, where $\left[D_{e}\right]$ is the elastic matrix and $\left\{\mathrm{d} \varepsilon^{p}\right\}$ is the plastic strain increment.

$\varepsilon_{v}, \bar{\varepsilon}$, and $\psi$ are three invariants of the strain tensor, where $\psi$ is the strain Lode's angle. If we ignore the effect of Lode's angle and take $\varepsilon_{v}$ and $\bar{\varepsilon}$ as potential functions, that is, $F_{1}=$ $\varepsilon_{v}, F_{2}=\bar{\varepsilon}$ in (19), then

$$
\mathrm{d} \sigma_{i j}^{p}=\lambda_{1} \frac{\partial \varepsilon_{v}}{\partial \varepsilon_{i j}}+\lambda_{2} \frac{\partial \bar{\varepsilon}}{\partial \varepsilon_{i j}}
$$

Written in matrix form,

$$
\left\{\mathrm{d} \sigma^{p}\right\}=\lambda_{1}\left\{\frac{\partial \varepsilon_{v}}{\partial \varepsilon}\right\}+\lambda_{2}\left\{\frac{\partial \bar{\varepsilon}}{\partial \varepsilon}\right\} .
$$

Obviously,

$$
\lambda_{1}=\mathrm{d} p^{p}, \quad \lambda_{2}=\mathrm{d} q^{p} .
$$

From the definition of (17),

$$
\begin{gathered}
\mathrm{d} p^{p}=K_{e} \mathrm{~d} \varepsilon_{v}^{p}=K_{e}\left(\mathrm{~d} \varepsilon_{v}-\mathrm{d} \varepsilon_{v}^{e}\right)=K_{e} \mathrm{~d} \varepsilon_{v}-\mathrm{d} p, \\
\mathrm{~d} q^{p}=3 G_{e} \mathrm{~d} \bar{\varepsilon}^{p}=3 G_{e}\left(\mathrm{~d} \bar{\varepsilon}-\mathrm{d} \bar{\varepsilon}^{e}\right)=3 G_{e} \mathrm{~d} \bar{\varepsilon}-\mathrm{d} q,
\end{gathered}
$$

where $K_{e}, G_{e}$ are the elastic bulk modulus and shear modulus. Considering

$$
\begin{aligned}
\mathrm{d} \varepsilon_{v}^{e} & =\frac{1}{K_{e}} \mathrm{~d} p, \\
\mathrm{~d} \bar{\varepsilon}^{e} & =\frac{1}{3 G_{e}} \mathrm{~d} q,
\end{aligned}
$$

substituting (39a) and (39b) into (27a) and (27b) yields

$$
\begin{gathered}
\mathrm{d} \varepsilon_{v}=\left(\frac{1}{K_{e}}+A\right) \mathrm{d} p+B \mathrm{~d} q, \\
\mathrm{~d} \bar{\varepsilon}=C \mathrm{~d} p+\left(\frac{1}{3 G_{e}}+D\right) \mathrm{d} q .
\end{gathered}
$$

It can now be calculated that

$$
\begin{aligned}
& \mathrm{d} p=\bar{A} \mathrm{~d} \varepsilon_{v}+\bar{B} \mathrm{~d} \bar{\varepsilon}, \\
& \mathrm{d} q=\bar{C} \mathrm{~d} \varepsilon_{v}+\bar{D} \mathrm{~d} \bar{\varepsilon},
\end{aligned}
$$

where

$$
\begin{gathered}
\bar{A}=\frac{1}{|A|}\left(D+\frac{1}{3 G_{e}}\right), \quad \bar{B}=-\frac{B}{|A|}, \quad \bar{C}=-\frac{C}{|A|}, \\
\bar{D}=\frac{1}{|A|}\left(A+\frac{1}{K_{e}}\right), \\
|A|=\frac{3 D G_{e}+A K_{e}+1}{3 K_{e} G_{e}}+(A D-B C) .
\end{gathered}
$$

Substituting (41a) and (41b) into (37), (38a), and (38b) gives

$$
\begin{gathered}
\lambda_{1}=\mathrm{d} p^{p}=\left(K_{e}-\bar{A}\right) \mathrm{d} \varepsilon_{v}-\bar{B} \mathrm{~d} \bar{\varepsilon} \\
\lambda_{2}=\mathrm{d} q^{p}=-\bar{C} \mathrm{~d} \varepsilon_{v}+\left(3 G_{e}-\bar{D}\right) \mathrm{d} \bar{\varepsilon} .
\end{gathered}
$$

In addition,

$$
\mathrm{d} \varepsilon_{v}=\left\{\frac{\partial \varepsilon_{v}}{\partial \varepsilon}\right\}^{T}\{\mathrm{~d} \varepsilon\}, \quad \mathrm{d} \bar{\varepsilon}=\left\{\frac{\partial \bar{\varepsilon}}{\partial \varepsilon}\right\}^{T}\{\mathrm{~d} \varepsilon\} .
$$

Substituting (44) into (43a), (43b), and (36) yields

$$
\left\{\mathrm{d} \sigma^{p}\right\}=\left[D_{p}^{\varepsilon}\right]\{\mathrm{d} \varepsilon\}
$$


where

$$
\begin{aligned}
{\left[D_{p}^{\varepsilon}\right]=} & \left(K_{e}-\bar{A}\right)\left\{\frac{\partial \varepsilon_{v}}{\partial \varepsilon}\right\}\left\{\frac{\partial \varepsilon_{v}}{\partial \varepsilon}\right\}^{T}-\bar{B}\left\{\frac{\partial \varepsilon_{v}}{\partial \varepsilon}\right\}\left\{\frac{\partial \bar{\varepsilon}}{\partial \varepsilon}\right\}^{T} \\
& -\bar{C}\left\{\frac{\partial \bar{\varepsilon}}{\partial \varepsilon}\right\}\left\{\frac{\partial \varepsilon_{v}}{\partial \varepsilon}\right\}^{T}+\left(3 G_{e}-\bar{D}\right)\left\{\frac{\partial \bar{\varepsilon}}{\partial \varepsilon}\right\}\left\{\frac{\partial \bar{\varepsilon}}{\partial \varepsilon}\right\}^{T},
\end{aligned}
$$

that is,

$$
\begin{aligned}
{\left[D_{p}^{\varepsilon}\right]=\frac{1}{|A|}\left\{\alpha A\left\{\frac{\partial \varepsilon_{v}}{\partial \varepsilon}\right\}\left\{\frac{\partial \varepsilon_{v}}{\partial \varepsilon}\right\}^{T}+B\left\{\frac{\partial \varepsilon_{v}}{\partial \varepsilon}\right\}\left\{\frac{\partial \bar{\varepsilon}}{\partial \varepsilon}\right\}^{T}\right.} \\
\left.+C\left\{\frac{\partial \bar{\varepsilon}}{\partial \varepsilon}\right\}\left\{\frac{\partial \varepsilon_{v}}{\partial \varepsilon}\right\}^{T}+\frac{D}{\alpha}\left\{\frac{\partial \bar{\varepsilon}}{\partial \varepsilon}\right\}\left\{\frac{\partial \bar{\varepsilon}}{\partial \varepsilon}\right\}^{T}\right\},
\end{aligned}
$$

where $\alpha=K_{e} / 3 G_{e}=2\left(1+\mu_{e}\right) / 9\left(1-2 \mu_{e}\right)$, and $\mu_{e}$ is the elastic Poisson ratio.

Hence, the total stress increment can be expressed as

$$
\begin{aligned}
\{\mathrm{d} \sigma\} & =\left[D_{e}\right]\left\{\mathrm{d} \varepsilon^{e}\right\}=\left[D_{e}\right]\left(\{\mathrm{d} \varepsilon\}-\left\{\mathrm{d} \varepsilon^{p}\right\}\right) \\
& =\left[D_{e}\right]\{\mathrm{d} \varepsilon\}-\left\{\mathrm{d} \sigma^{p}\right\}=\left[D_{e}\right]\{\mathrm{d} \varepsilon\}-\left[D_{p}^{\varepsilon}\right]\{\mathrm{d} \varepsilon\} \\
& =\left[D_{e p}^{\varepsilon}\right]\{\mathrm{d} \varepsilon\},
\end{aligned}
$$

where

$$
\left[D_{e p}^{\varepsilon}\right]=\left[D_{e}\right]-\left[D_{p}^{\varepsilon}\right] .
$$

The duality of stress and strain is evident in (45a), (45b), (45c), (33a), and (33b).

It should be noted that it is practically impossible to obtain the total strain in soil, and thus the elastoplastic matrix in stress space is more applicable. However, if we could further extend the framework to the space of strain increment, the practicability becomes promising.

\section{Relationship with Traditional Elastoplastic Model}

7.1. General Form. The elastoplastic compliance matrix of the traditional elastoplastic model is

$$
\left[C_{e p}^{\sigma}\right]=\left[C_{e}\right]+\frac{1}{A_{H}}\left\{\frac{\partial g}{\partial \sigma}\right\}\left\{\frac{\partial f}{\partial \sigma}\right\}^{T},
$$

where $f$ and $g$ are the yield function and plastic potential function, $A_{H}=-\partial f / \partial H\left\{\partial H / \partial \varepsilon^{p}\right\}^{T}\{\partial g / \partial \sigma\}$ is the plastic hardening modulus, and $H$ is the hardening parameter.

$f$ and $g$ are usually expressed in terms of the stress invariants, $p, q, \theta$. If the effect of the Lode's angle $\theta$ is not considered, the expression only concerns $p$ and $q$, that is,

$$
\begin{aligned}
& \left\{\frac{\partial f}{\partial \sigma}\right\}=\frac{\partial f}{\partial p}\left\{\frac{\partial p}{\partial \sigma}\right\}+\frac{\partial f}{\partial q}\left\{\frac{\partial q}{\partial \sigma}\right\}, \\
& \left\{\frac{\partial g}{\partial \sigma}\right\}=\frac{\partial g}{\partial p}\left\{\frac{\partial p}{\partial \sigma}\right\}+\frac{\partial g}{\partial q}\left\{\frac{\partial q}{\partial \sigma}\right\} .
\end{aligned}
$$

Substituting (48a) and (48b) into (47), we have

$$
\begin{aligned}
& {\left[C_{e p}^{\sigma}\right]} \\
& =\left[C_{e}\right]+\frac{1}{A_{H}} \\
& \quad \times\left\{\frac{\partial g}{\partial p} \frac{\partial f}{\partial p}\left\{\frac{\partial p}{\partial \sigma}\right\}\left\{\frac{\partial p}{\partial \sigma}\right\}^{T}+\frac{\partial g}{\partial p} \frac{\partial f}{\partial q}\left\{\frac{\partial p}{\partial \sigma}\right\}\left\{\frac{\partial q}{\partial \sigma}\right\}^{T}\right. \\
& \left.+\frac{\partial g}{\partial q} \frac{\partial f}{\partial p}\left\{\frac{\partial q}{\partial \sigma}\right\}\left\{\frac{\partial p}{\partial \sigma}\right\}^{T}+\frac{\partial g}{\partial q} \frac{\partial f}{\partial q}\left\{\frac{\partial q}{\partial \sigma}\right\}\left\{\frac{\partial q}{\partial \sigma}\right\}^{T}\right\} .
\end{aligned}
$$

Comparing this with (33a), (33b), (34a), and (34b), it follows that

$$
\begin{array}{ll}
A=\frac{1}{A_{H}} \frac{\partial g}{\partial p} \frac{\partial f}{\partial p}, & B=\frac{1}{A_{H}} \frac{\partial g}{\partial p} \frac{\partial f}{\partial q}, \\
C=\frac{1}{A_{H}} \frac{\partial g}{\partial q} \frac{\partial f}{\partial p}, & D=\frac{1}{A_{H}} \frac{\partial g}{\partial q} \frac{\partial f}{\partial q} .
\end{array}
$$

Equation (33a) and (33b) can be seen as a general formula for traditional constitutive models, and (49) is a special form of (33a) and (33b). For associated models, when $f=g$,

$$
\begin{gathered}
A=\frac{1}{A_{H}}\left(\frac{\partial f}{\partial p}\right)^{2}, \quad B=C=\frac{1}{A_{H}} \frac{\partial f}{\partial p} \frac{\partial f}{\partial q}, \\
D=\frac{1}{A_{H}}\left(\frac{\partial f}{\partial q}\right)^{2} .
\end{gathered}
$$

Most traditional constitutive models are to determine the relationship between $f, g$ and $p, q$, which can be used to calculate the four model parameters $A, B, C$, and $D$ indirectly. It can be seen from (50a) and (50b) that the traditional nonassociated models actually assume that

$$
A D-B C=0 .
$$

The associated models still need to satisfy (51a) and also require

$$
B=C \text {. }
$$

Rewrite (27a) and (27b) into matrix form

$$
\left\{\begin{array}{l}
\mathrm{d} \varepsilon_{v}^{p} \\
\mathrm{~d} \bar{\varepsilon}^{p}
\end{array}\right\}=\left[\begin{array}{ll}
A & B \\
C & D
\end{array}\right]\left\{\begin{array}{l}
\mathrm{d} p \\
\mathrm{~d} q
\end{array}\right\} .
$$

Clearly (51a) requires the determinant rank of the coefficient matrix in (52) to be 1 or requires $\mathrm{d} \varepsilon_{v}^{p}$ and $\mathrm{d} \bar{\varepsilon}^{p}$ to be linearly correlated. Equation (51b) additionally requires the coefficient matrix to be symmetric.

The traditional elastoplastic model in stress space can be translated to strain space by the duality of (45a), (45b), (45c), 
(33a), and (33b). Substituting (50a) and (50b) into (42), (45a), (45b), and (45c) yields the expression in strain space

$$
\begin{aligned}
& {\left[D_{e p}^{\varepsilon}\right]} \\
& =\left[D_{e}\right]-\frac{1}{\beta} \\
& \quad \times\left\{\alpha \frac{\partial g}{\partial p} \frac{\partial f}{\partial p}\left\{\frac{\partial \varepsilon_{v}}{\partial \varepsilon}\right\}\left\{\frac{\partial \varepsilon_{v}}{\partial \varepsilon}\right\}^{T}+\frac{\partial g}{\partial p} \frac{\partial f}{\partial q}\left\{\frac{\partial \varepsilon_{v}}{\partial \varepsilon}\right\}\left\{\frac{\partial \bar{\varepsilon}}{\partial \varepsilon}\right\}^{T}\right. \\
& \left.+\frac{\partial g}{\partial q} \frac{\partial f}{\partial p}\left\{\frac{\partial \varepsilon_{v}}{\partial \varepsilon}\right\}\left\{\frac{\partial \bar{\varepsilon}}{\partial \varepsilon}\right\}^{T}+\frac{1}{\alpha} \frac{\partial g}{\partial q} \frac{\partial f}{\partial q}\left\{\frac{\partial \bar{\varepsilon}}{\partial \varepsilon}\right\}\left\{\frac{\partial \bar{\varepsilon}}{\partial \varepsilon}\right\}^{T}\right\},
\end{aligned}
$$

where

$$
\beta=A_{H}|A|=\frac{1}{3 G_{e}} \frac{\partial g}{\partial p} \frac{\partial f}{\partial p}+\frac{1}{K_{e}} \frac{\partial g}{\partial q} \frac{\partial f}{\partial q}+\frac{A_{H}}{3 K_{e} G_{e}} .
$$

Hence, the stiffness matrix of (53a) and (53b) is obtained using $f$ and $g$ from the traditional constitutive models, and the transformation from stress space to strain space is thus achieved. Obviously, the transformation only changes the mathematical calculation method of the coefficient and elastoplastic matrices and has no influence on a particular model itself or the loading-unloading criterion of the model. Therefore, the transformation is applicable to all traditional elastoplastic models.

7.2. Modified Cambridge Model. In the modified Cambridge model,

$$
f=g=p+\frac{q^{2}}{M^{2} p}-p_{0} e^{\left(1+e_{0}\right) /(\lambda-\kappa) H}=0,
$$

and so

$$
\begin{gathered}
\frac{\partial f}{\partial p}=1-\frac{\eta^{2}}{M^{2}}, \quad \frac{\partial f}{\partial q}=\frac{2 \eta}{M^{2}}, \\
A_{H}=\frac{1+e_{0}}{\lambda-\kappa}\left(1-\frac{\eta^{2}}{M^{2}}\right) p_{0} e^{\left(1+e_{0}\right) /(\lambda-\kappa) H},
\end{gathered}
$$

where $\eta=q / p, H$ is the hardening parameter $\left(=\varepsilon_{v}^{p}\right.$, for the modified Cambridge model), $M$ is the stress ratio at critical state, $p_{0}$ is the initial mean stress, $e_{0}$ is the initial void ratio, $\lambda$ is the slope of the normal compression line (NCL), and $\kappa$ is the slope of the unloading line. The elastoplastic compliance matrix in stress space is expressed as

$$
\begin{aligned}
{\left[C_{e p}^{\sigma}\right]=\left[C_{e}\right] } & +\frac{1}{A_{H}} \\
& \times\left\{\left(1-\frac{\eta^{2}}{M^{2}}\right)^{2}\left\{\frac{\partial p}{\partial \sigma}\right\}\left\{\frac{\partial p}{\partial \sigma}\right\}^{T}\right. \\
& +\frac{2 \eta}{M^{2}}\left(1-\frac{\eta^{2}}{M^{2}}\right)\left\{\frac{\partial p}{\partial \sigma}\right\}\left\{\frac{\partial q}{\partial \sigma}\right\}^{T} \\
& +\frac{2 \eta}{M^{2}}\left(1-\frac{\eta^{2}}{M^{2}}\right)\left\{\frac{\partial q}{\partial \sigma}\right\}\left\{\frac{\partial p}{\partial \sigma}\right\}^{T} \\
& \left.+\frac{4 \eta^{2}}{M^{4}}\left\{\frac{\partial q}{\partial \sigma}\right\}\left\{\frac{\partial q}{\partial \sigma}\right\}^{T}\right\} .
\end{aligned}
$$

The elastoplastic stiffness matrix in strain space is

$$
\begin{aligned}
{\left[D_{e p}^{\varepsilon}\right]=\left[D_{e}\right]-\frac{1}{\beta} } & \\
\times & \left\{\frac{K_{e}}{3 G_{e}}\left(1-\frac{\eta^{2}}{M^{2}}\right)^{2}\left\{\frac{\partial \varepsilon_{v}}{\partial \varepsilon}\right\}\left\{\frac{\partial \varepsilon_{v}}{\partial \varepsilon}\right\}^{T}\right. \\
& +\frac{2 \eta}{M^{2}}\left(1-\frac{\eta^{2}}{M^{2}}\right)\left\{\frac{\partial \varepsilon_{v}}{\partial \varepsilon}\right\}\left\{\frac{\partial \bar{\varepsilon}}{\partial \varepsilon}\right\}^{T} \\
& +\frac{2 \eta}{M^{2}}\left(1-\frac{\eta^{2}}{M^{2}}\right)\left\{\frac{\partial \varepsilon_{v}}{\partial \varepsilon}\right\}\left\{\frac{\partial \bar{\varepsilon}}{\partial \varepsilon}\right\}^{T} \\
& \left.+\frac{3 G_{e}}{K_{e}} \frac{4 \eta^{2}}{M^{4}}\left\{\frac{\partial \bar{\varepsilon}}{\partial \varepsilon}\right\}\left\{\frac{\partial \bar{\varepsilon}}{\partial \varepsilon}\right\}^{T}\right\},
\end{aligned}
$$

where

$$
\beta=\frac{1}{3 G_{e}}\left(1-\frac{\eta^{2}}{M^{2}}\right)^{2}+\frac{1}{K_{e}} \frac{4 \eta^{2}}{M^{4}}+\frac{A_{H}}{3 G_{e} K_{e}} .
$$

A new hardening parameter for the modified Cambridge model was proposed by Yao et al. [12] as

$$
H=\int \mathrm{d} H=\int \frac{M_{f}^{4}-\eta^{4}}{M^{4}-\eta^{4}} \mathrm{~d} \varepsilon_{v}^{p}=\int \frac{1}{\Omega} \mathrm{d} \varepsilon_{v}^{p} .
$$

in which

$$
\Omega=\frac{M^{4}-\eta^{4}}{M_{f}^{4}-\eta^{4}}
$$

where $M_{f}$ is the potential failure stress ratio.

Yao improved the modified Cambridge model by replacing $H=\varepsilon_{v}^{p}$ with (58a) and (58b), which changes $A_{H}$ in the modified Cambridge model to $(1 / \Omega) A_{H}$. The improved constitutive model is a unified hardening model and is suitable for sandy soil, which actually replaces $\left\{\mathrm{d} \varepsilon^{p}\right\}$ by $\Omega\left\{\mathrm{d} \varepsilon^{p}\right\}$ with (49), or the following expression with (52):

$$
\frac{\mathrm{d} \varepsilon_{v}^{p}}{\left.\mathrm{~d} \varepsilon_{v}^{p}\right|_{c}}=\frac{\mathrm{d} \bar{\varepsilon}^{p}}{\left.\mathrm{~d} \bar{\varepsilon}^{p}\right|_{c}}=\Omega=\frac{M^{4}-\eta^{4}}{M_{f}^{4}-\eta^{4}}
$$

where $\left.\mathrm{d} \varepsilon_{v}^{p}\right|_{c},\left.\mathrm{~d} \bar{\varepsilon}^{p}\right|_{c}$ are the volumetric strain and shear strain, respectively, that are calculated by the modified Cambridge model.

This modification can be further improved. For instance, the volumetric strain and shear strain of triaxial testing are first calculated by the modified Cambridge model, and then the ratio of the volumetric strain and shear strain can be fitted according to the test results, that is

$$
\frac{\mathrm{d} \varepsilon_{v}^{p}}{\left.\mathrm{~d} \varepsilon_{v}^{p}\right|_{c}}=\xi(p, q), \quad \frac{\mathrm{d} \bar{\varepsilon}^{p}}{\left.\mathrm{~d} \bar{\varepsilon}^{p}\right|_{c}}=\zeta(p, q) .
$$

Therefore, (52) is modified to

$$
\left\{\begin{array}{l}
\mathrm{d} \varepsilon_{v}^{p} \\
\mathrm{~d} \bar{\varepsilon}^{p}
\end{array}\right\}=\left[\begin{array}{ll}
\xi A & \xi B \\
\zeta C & \zeta D
\end{array}\right]\left\{\begin{array}{l}
\mathrm{d} p \\
\mathrm{~d} q
\end{array}\right\} .
$$

$\xi$ and $\zeta$ can be estimated by polynomial fitting or other fitting methods. Yao's hardening model is obtained when $\xi=$ $\zeta=\left(M^{4}-\eta^{4}\right) /\left(M_{f}^{4}-\eta^{4}\right)$. 
The above-mentioned modification method can be extended to other elastoplastic models. The correction coefficients $\xi$ and $\zeta$ can be fitted based on the triaxial testing results or other testing results. The obtained matrix can improve the calculation accuracy of existing elastoplastic models or be used to establish new modified models. Note that there is no physical mechanism involved in the framework, and thus the loading-unloading criterion and the evolution of internal state variables of the original model should still be employed in the modified one.

\section{Application: A Simple Model}

Theoretically, the constitutive model of soil would be established if the parameters $A, B, C$, and $D$ in (27a), (27b), (33a), and (33b) are obtained by experiments such as conventional triaxial test, isotropic compression test, and $p=$ Const. test.

For example, the equations for the tangent modulus $E_{t}$ and tangential Poisson ratio $\mu_{t}$ are obtained by fitting the curve from triaxial testing, that is

$$
\begin{gathered}
E_{t}=\frac{\partial\left(\sigma_{1}-\sigma_{3}\right)}{\partial \varepsilon_{1}}, \\
\mu_{t}=-\frac{\partial \varepsilon_{3}}{\partial \varepsilon_{1}}=\frac{1}{2}\left(1-\frac{\partial \varepsilon_{v}}{\partial \varepsilon_{1}}\right) .
\end{gathered}
$$

$E_{t}$ and $\mu_{t}$ can be curve fitted by a polynomial or by the formulae in the Duncan-Chang E- $\mu$ model [1]. However, two supplementary equations are needed as there are four unknown parameters in (27a) and (27b). Therefore, an isotropic compression test or $p=$ Const. test should be conducted, or the assumptions $A D-B C=0$ and $B=C$ are made.

In conventional triaxial test, $\sigma_{3}=$ Const, $\mathrm{d} p=(1 / 3) \mathrm{d} \sigma_{1}$, $\mathrm{d} q=\mathrm{d} \sigma_{1}, \mathrm{~d} \varepsilon_{v}=\mathrm{d} \varepsilon_{1}+\mathrm{d} \varepsilon_{2}+\mathrm{d} \varepsilon_{3}=\left(1-2 \mu_{t}\right) \mathrm{d} \varepsilon_{1}$, and $\mathrm{d} \bar{\varepsilon}=$ $(2 / 3)\left(1+\mu_{t}\right) \mathrm{d} \varepsilon_{1}$. According to (62a), (62b), (27a), and (27b) and the assumption in (51a) and (51b), we have

$$
A=\frac{K_{e p}^{2}}{\omega}, \quad B=C=\frac{K_{e p} G_{e p}}{\omega}, \quad D=\frac{G_{e p}^{2}}{\omega},
$$

where

$$
\begin{aligned}
K_{e p} & =\frac{1-2 \mu_{t}}{E_{t}}-\frac{1-2 \mu_{e}}{E_{e}}, \\
G_{e p} & =\frac{2\left(1+\mu_{t}\right)}{3 E_{t}}-\frac{2\left(1+\mu_{e}\right)}{3 E_{e}}, \\
\omega & =G_{e p}+\frac{1}{3} K_{e p}=\frac{1}{E_{t}}-\frac{1}{E_{e}} .
\end{aligned}
$$

$E_{e}$ is the elastic modulus, and the elastic Poisson ratio $\mu_{e}$ is generally taken as 0.3 for soil. It is obvious that $\omega>0$ is always fulfilled in (63a) and (63b).

By substituting (63a) and (63b) into (33a), (33b), or (35), the elastoplastic compliance matrix in stress space or the stiffness matrix in strain space is obtained. For convenience, we call this model the multiple potential surface model (MPS model).

It should be noted that, contrary to the Duncan-Chang model (DC model), $E_{t}$ and $\mu_{t}$ in the MPS model are not

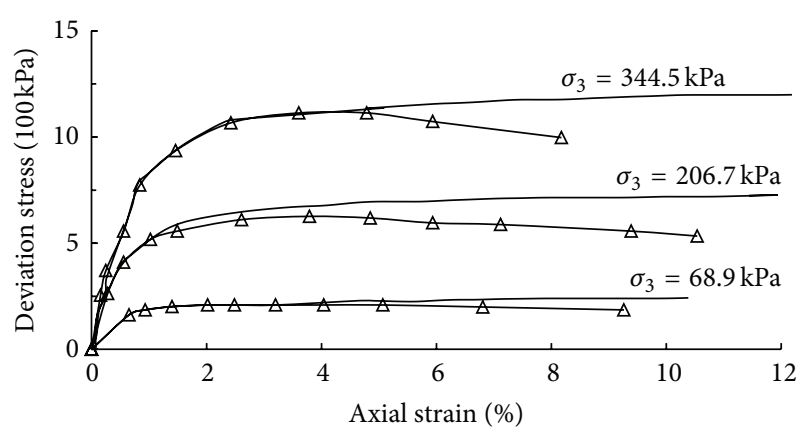

- Calculation result
$\longleftarrow \quad$ Experimental result

(a)

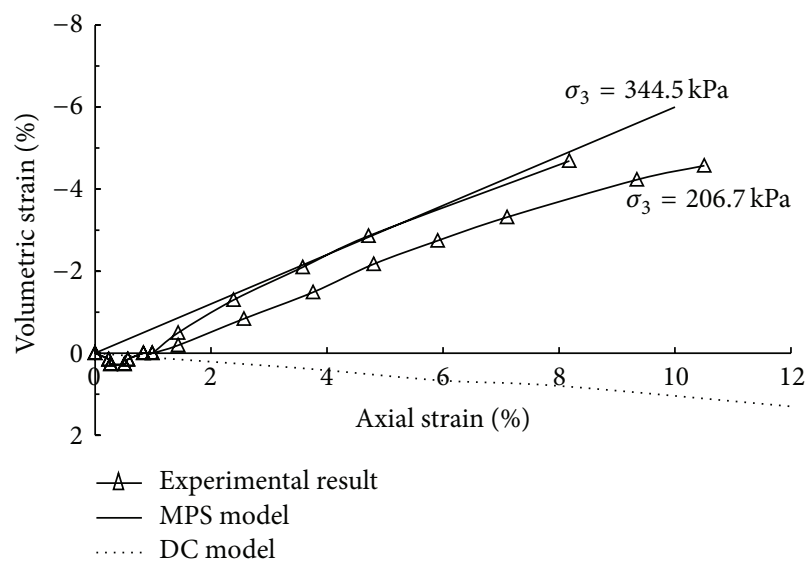

(b)

FIGURE 1: Calculation and test results for triaxial test of Ottawa silica sand.

limited by the generalized Hooke's law; that is, the MPS model is still available when $\mu_{t}>0.5$ and the stiffness matrix of the model is not singular. Actually, $E_{t}$ and $\mu_{t}$ in the new model are not the traditional modulus and Poisson ratio, but just the slope of the curves.

Figure 1 shows the calculation and test results for triaxial testing of Ottawa silica sand conducted by $\mathrm{Wu}$ [37]. The unit weight of the sand is $16.8 \mathrm{kN} / \mathrm{m}^{3}$ (=107 pcf). The test is a conventional consolidated-drained triaxial compression test (CD test). The confining pressures were 68.9, 206.7, and $344.5 \mathrm{kPa}(=10,30$, and $50 \mathrm{psi}$, resp.). During the CD test, confining pressure was firstly applied and then the specimen was consolidated. Deviation stress $\left(\sigma_{1}-\sigma_{3}\right)$ was applied in the axial direction after consolidation. Variations of deviation stress and volumetric strain versus axial strain can be acquired in the test.

The calculations were made using the MPS model as well as the Duncan-Chang model, during which $E_{t}, E_{e}$, and $\mu_{t}$ were calculated by the method proposed by Duncan and Chang [1], that is

$$
\begin{gathered}
E_{t}=\left[1-\frac{R_{f}(1-\sin \phi)\left(\sigma_{1}-\sigma_{3}\right)}{2 c \cos \phi+2 \sigma_{3} \sin \phi}\right]^{2} K P_{a}\left(\frac{\sigma_{3}}{P_{a}}\right)^{n}, \\
E_{e}=K_{u r} P_{a}\left(\frac{\sigma_{3}}{P_{a}}\right)^{n},
\end{gathered}
$$




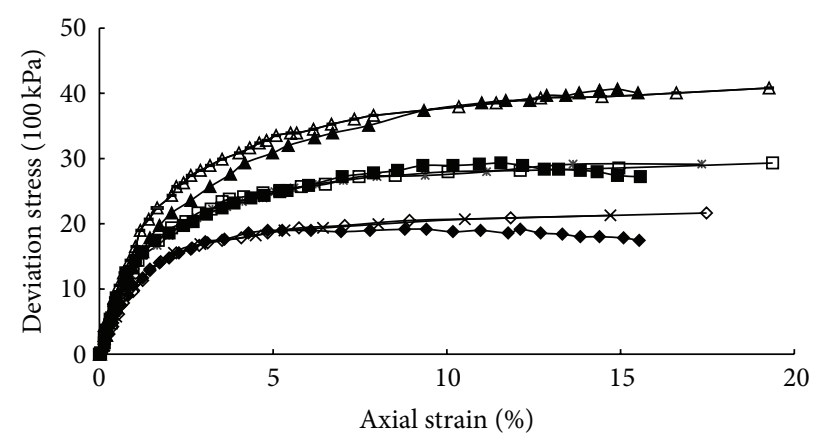

(a)

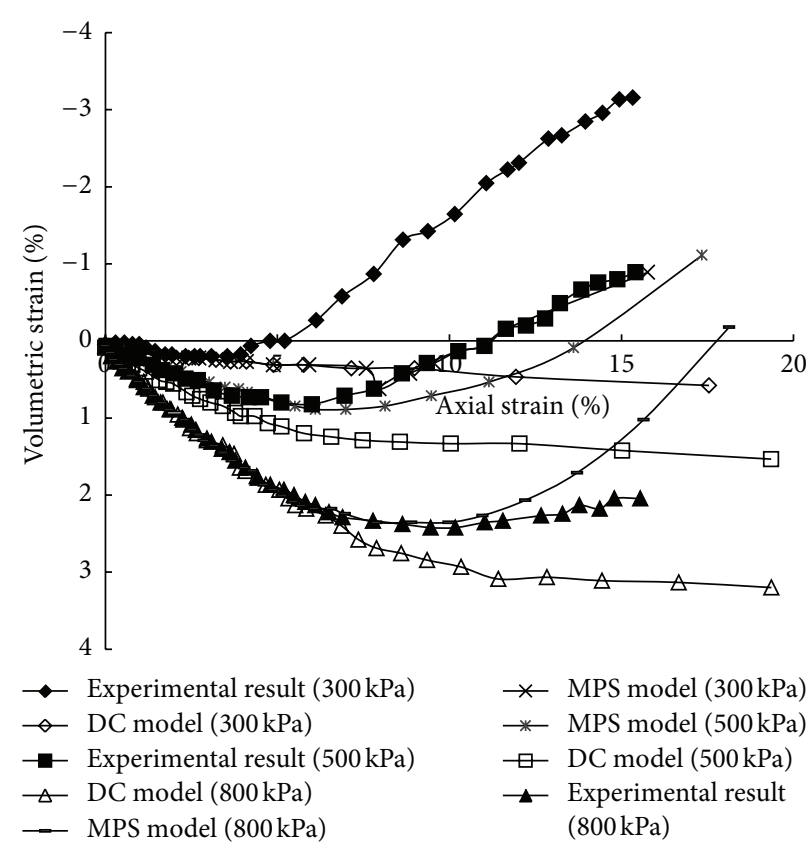

(b)

FIGURE 2: Calculation and test results for triaxial test of rockfill material.

$$
\begin{gathered}
\mu_{t}=\frac{G-F l g\left(\sigma_{3} / P_{a}\right)}{\left(1-A^{*}\right)^{2}}, \\
A^{*}=\frac{D\left(\sigma_{1}-\sigma_{3}\right)}{K P_{a}\left(\sigma_{3} / P_{a}\right)^{n}\left[1-R_{f}(1-\sin \phi)\left(\sigma_{1}-\sigma_{3}\right) /\left(2 c \cos \phi+2 \sigma_{3} \sin \phi\right)\right]},
\end{gathered}
$$

where $c$ is the cohesion of the soil, $\phi$ is the friction angle of the soil, $P_{a}$ is the atmospheric pressure, $100 \mathrm{kPa} K K, K_{u r}, n$, $R_{f}, G, F$, and $D$ are parameters.

The parameters in the calculation are taken as $c=0 \mathrm{kPa}$, $\phi=38^{\circ}, K=1116, K_{u r}=1500, n=0.65, R_{f}=0.88, F=0$, and $D=0$, which are the same for both the DC and MPS models. The value of $G$ for the $D C$ model is taken as 0.45 while for the MPS model it is 0.8 , which is larger than 0.5. Although the calculation results for deviation stress are identical for the two models, the MPS model can reproduce the dilation of soil. Because of the limitation that $\mu_{t}<0.5$ in the DC model, the dilatation of soil is not revealed and $\varepsilon_{v}<0$ is not achieved.

Figure 2 shows the calculation and test results of consolidated-drained triaxial compression test (CD test) of a rock-fill material from Hengshan Dam in China. The unit weight of the material is $20.7 \mathrm{kN} / \mathrm{m}^{3}$. The confining pressures were 300,500 , and $800 \mathrm{kPa}$, respectively.

The parameters for $E_{t}$ are $c=178 \mathrm{kPa}, \phi=40.4^{\circ}, K=$ $1915, K_{u r}=2490, n=0.18$, and $R_{f}=0.85$, which are also the same for both the DC and MPS models. $\mu_{t}$ in the DC model is still calculated using (65a) and (65b), and $G=0.6, F=0.37$, and $D=0.023$ in the calculation, while $\mu_{t}$ in the MPS model is calculated using the method proposed by Shen and Zhang [38],

$$
\begin{gathered}
\mu_{t}=\frac{1}{2}-c_{d}\left(\frac{\sigma_{3}}{P_{a}}\right)^{n_{d}} \frac{E_{i} R_{f}}{\left(\sigma_{1}-\sigma_{3}\right)_{f}} \frac{1-R_{d}}{R_{d}} \\
\times\left(1-\frac{R_{f} S_{l}}{1-R_{f} S_{l}} \frac{1-R_{d}}{R_{d}}\right), \\
E_{i}=K P_{a}\left(\frac{\sigma_{3}}{P_{a}}\right)^{n},
\end{gathered}
$$

in which $S_{l}=\left(\sigma_{1}-\sigma_{3}\right) /\left(\sigma_{1}-\sigma_{3}\right)_{f} ;\left(\sigma_{1}-\sigma_{3}\right)_{f}$ is the deviation stress at failure, $\left(2 c \cos \phi+2 \sigma_{3} \sin \phi\right) /(1-\sin \phi) ; c_{d}, n_{d}$, and $R_{d}$ are parameters; $c_{d}=0.000224, n_{d}=2.24$, and $R_{d}=0.85$ in the calculation.

Again, the calculation results for deviation stress are identical for the two models, while the MPS model reproduces the dilatation of soil giving better results than the DC model. Obviously, more appropriate results of volumetric strain can be acquired using the MPS model if we improve the calculation method of $\mu_{t}$. However, this is impossible for the DC model due to the limitation that $\mu_{t}$ cannot exceed 0.5 for the nonlinear elastic model. 


\section{Conclusions}

The main tasks in establishing the constitutive equations for geomaterials are the determination of stress-strain relations in principal stress/strain space and the coordinate transformation of the relationship from principal stress/strain space to general coordinate space. The stress (or strain) and stress increment (or strain increment) in principal stress/strain space is expressed as a vector in a potential field in traditional elastic potential theory and plastic potential theory. However, the vector can be expressed more generally as the gradient vector of linearly independent potential functions. Based on this framework, the traditional models can be easily transformed from stress space to strain space and can be modified in a general way. This framework can also be used to establish new models based on curve fitting. Since it investigates constitutive models from a mathematical point of view independent of the material itself and relevant physical mechanism, the framework can be potentially used in a wider range, not limited to geomaterials. However, the lack of physical insights of materials and constitutive models may also hinder its development, for example, the constitutive model based on the framework may be oversimplified. The loadingunloading criteria as well as the evolutions of internal state variables are not considered in the framework. Therefore, the current framework is mainly useful for modifying the existing models. In the next research, Lode's angle and noncoaxiality should also be investigated, and more test results are needed to make the verification.

\section{Acknowledgments}

The support of the Natural Science Foundation of China (51279085), National Basic Research Program of China (973 Program 2013CB036402), and the State Key Laboratory of Hydroscience and Engineering (sklhse-2012-D-01) is gratefully acknowledged.

\section{References}

[1] J. M. Duncan and C. Y. Chang, "Nonlinear analysis of stress and strain in soils," ASCE Journal of the Soil Mechanics and Foundations Division, vol. 96, no. 5, pp. 1629-1653, 1970.

[2] J. M. Duncan, P. Byren, K. Wong, and P. Mabry, "Strength, stress-strain and bulk modulous parametres for finite element analysis of stress and movements in soils masses," Tech. Rep. UCB/GT/80O01, University of California, Berkeley, Calif, USA, 1980.

[3] D. Battelino and B. Majes, "A hypoelastic model of soils accounting for failure," in Proceedings of the 9th International Conference on Soil Mechanics and Foundation Engineering, pp. 39-42, Tokyo, Japan, 1977.

[4] L. Domaschuk and P. Valliappan, "Nonlinear settlement analysis by finite element," ASCE Journal of the Geotechnical Engineering Division, vol. 101, no. 7, pp. 601-614, 1975.

[5] D. J. Naylor, "Stress-strain laws for soils," in Developments in Soil Mechanics, C. R. Scott, Ed., chapter 2, pp. 39-68, Applied Science, London, UK, 1978.

[6] D. C. Drucker, R. E. Gibson, and D. J. Henkek, "Soil mechanics and work hardening theories of plasticity," ASCE Journal of the
Soil Mechanics and Foundations Division, vol. 81, no. 1, pp. 1-14, 1955.

[7] K. H. Roscoe, A. N. Schofield, and A. Thurairajah, "Yielding of clay in states wetter than critical," Geotechnique, vol. 13, no. 3, pp. 211-240, 1963.

[8] K. H. Roscoe and J. B. Burland, "On the generalised stressstrain behaviour of an ideal wet clay," in Engineering Plasticity , J. Heyman and F. A. Leckie, Eds., pp. 535-609, Cambridge University Press, Cambridge, UK, 1968.

[9] C. P. Wroth and R. H. Bassett, "A stress-strain relationship for the shearing behavior of sand," Geotechnique, vol. 15, no. 1, pp. 32-56, 1965.

[10] H. B. Poorooshasb, I. Holubec, and A. N. Sherbome, "Yielding and flow of sand in triaxial compression," Canadian Geotechnical Journal, vol. 3, no. 4, pp. 179-190, 1966.

[11] Y. P. Yao, T. Luo, D. A. Sun, and H. Matsuoka, "A simple 3-D constitutive model for both clay and sand," Chinese Journal of Geotechnical Engineering, vol. 24, no. 2, pp. 240-246, 2002.

[12] Y.-P. Yao, W. Hou, and A.-N. Zhou, "UH model: threedimensional unified hardening model for overconsolidated clays," Geotechnique, vol. 59, no. 5, pp. 451-469, 2009.

[13] C. S. Desai and M. O. Faruque, "Constitutive model for geological materials," Journal of Engineering Mechanics, vol. 110, no. 9, pp. 1391-1408, 1984.

[14] C. S. Desai, S. Somasundaram, and G. Frantziskonis, "A hierarchical approach for constitutive modelling of geologic materials," International Journal for Numerical and Analytical Methods in Geomechanics, vol. 10, no. 3, pp. 225-257, 1986.

[15] P. V. Lade and J. M. Duncan, "Elasto-plastic stress-strain theory for cohesionless soil with curved yield surfaces," International Journal of Solids and Structures, vol. 13, no. 11, pp. 1019-1035, 1977.

[16] M. K. Kim and P. V. Lade, "Single hardening constitutive model for frictional materials. I. Plastic potential function," Computers and Geotechnics, vol. 5, no. 4, pp. 307-324, 1988.

[17] P. V. Lade and M. K. Kim, "Single hardening constitutive model for frictional materials II. Yield critirion and plastic work contours," Computers and Geotechnics, vol. 6, no. 1, pp. 13-29, 1988.

[18] P. V. Lade and M. K. Kim, "Single hardening constitutive model for frictional materials III. Comparisons with experimental data," Computers and Geotechnics, vol. 6, no. 1, pp. 31-47, 1988.

[19] Y. R. Zheng and D. J. Yan, "Multi-yield surface model for soils on the basis of test fitting," in Computer Methods and Advances in Geotechnics, pp. 97-104, Taylor \& Francis, Boca Raton, Fla, USA, 1994.

[20] Y. F. Dafalias and L. R. Herrmann, "Bounding surface soil plasticity model," Microstructural Science, vol. 1, pp. 335-345, 1980.

[21] Y. F. Dafalias, "Bounding surface plasticity. I: mathematical foundation and hypoplasticity," Journal of Engineering Mechanics, vol. 112, no. 9, pp. 966-987, 1986.

[22] Y. Dafalias and L. R. Hermann, "Bounding surface plasticity. II: application to isotropic cohesive soils," Journal of Engineering Mechanics, vol. 112, no. 12, pp. 1263-1291, 1986.

[23] A. Anandarajah and Y. F. Dafalias, "Bounding surface plasticity. III: application to anisotropic cohesive soils," Journal of Engineering Mechanics, vol. 112, no. 12, pp. 1292-1318, 1986.

[24] K. Hashiguchi, "Subloading surface model in unconventional plasticity," International Journal of Solids and Structures, vol. 25, no. 8, pp. 917-945, 1989. 
[25] K. Hashiguchi and Z.-P. Chen, "Elastoplastic constitutive equation of soils with the subloading surface and the rotational hardening," International Journal for Numerical and Analytical Methods in Geomechanics, vol. 22, no. 3, pp. 197-227, 1998.

[26] Z. P. Bazant, "Endochronic inelasticity and incremental plasticity," International Journal of Solids and Structures, vol. 14, no. 9, pp. 691-714, 1978.

[27] C. S. Desai and J. Toth, "Disturbed state constitutive modeling based on stress-strain and nondestructive behavior," International Journal of Solids and Structures, vol. 33, no. 11, pp. 16191650, 1996.

[28] G. H. Yang, "Building the generalized plastic potential theory in constitutive relation of soils," in Proceedings of the 3rd National Conference on the Numerical Analysis and Analytical Method for Rock and Soil Mechanics, pp. 140-148, Zhuhai, China, 1988.

[29] G. H. Yang, "A law for the general form of tensor in the constitutive equation of the soil and rock materials," in Proceedings of the Theory and Application of Water Power and Structure Engineering, pp. 315-321, Dalian, China, 1993.

[30] G. H. Yang, "Study on the mathematical constitutive theory of soils," in Proceedings of the 3rd National Young Scholar Conference on Rock and Soil Mechanics Engineering, pp. 100-112, Dalian, China, 1995.

[31] G. H. Yang, "A new strain space elasto-plastic constitutive model for soils," in Proceedings of the 2nd international conference on soft soil engineering, pp. 255-262, Nanjing, China, 1996.

[32] W. Zhou, Y. Liu, and J. Zhao, "Multi-potential based discontinuous bifurcation model for jointed rock masses and its application," Computer Methods in Applied Mechanics and Engineering, vol. 192, no. 33-34, pp. 3569-3584, 2003.

[33] A. Zhou and T. Lu, "Elasto-plastic constitutive model of soilstructure interface in consideration of strain softening and dilation," Acta Mechanica Solida Sinica, vol. 22, no. 2, pp. 171179, 2009.

[34] A. Anandarajah, K. Sobhan, and N. Kuganenthira, "Incremental stress-strain behavior of granular soil," ASCE Journal of the Geotechnical Engineering Division, vol. 121, no. 1, pp. 57-68, 1995.

[35] H. Matsuoka and T. Nakai, "Stress-strain relationship of soil based on the 'SMP"' in Proceedings of the 9th International Conference on Soil Mechanics and Foundation Engineering, pp. 153-162, Tokyo, Japan, 1977.

[36] T. Nakai and H. Matsuoka, "Constitutive equation for soils based on the extended concept of "Spatial Mobilized Plane" and its application to finite element analysis," Soils and Foundations, vol. 23, no. 4, pp. 87-105, 1983.

[37] J. T. H. Wu, "Predicting performance of the Denver walls: general report," in Proceedings of the International Symposium on Soft Geosynthetic-Reinforced Soil Retaining Walls, Balkema A. A., Tokyo, Japan, November 1992.

[38] Z. J. Shen and W. M. Zhang, "Analysis of effective stress and movements of Lubuge dam," in Numerical Methods in Geomechanics: Innsbruck 1988, Balkema A.A., Rotterdam, The Netherlands, 1988, Proceeding of the 6th International Conference on Numerical Methods in Geomechanics, Innsbruck, Austria. 


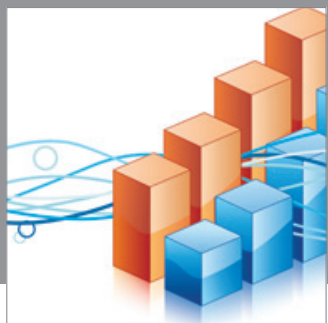

Advances in

Operations Research

mansans

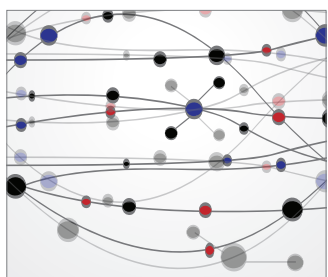

The Scientific World Journal
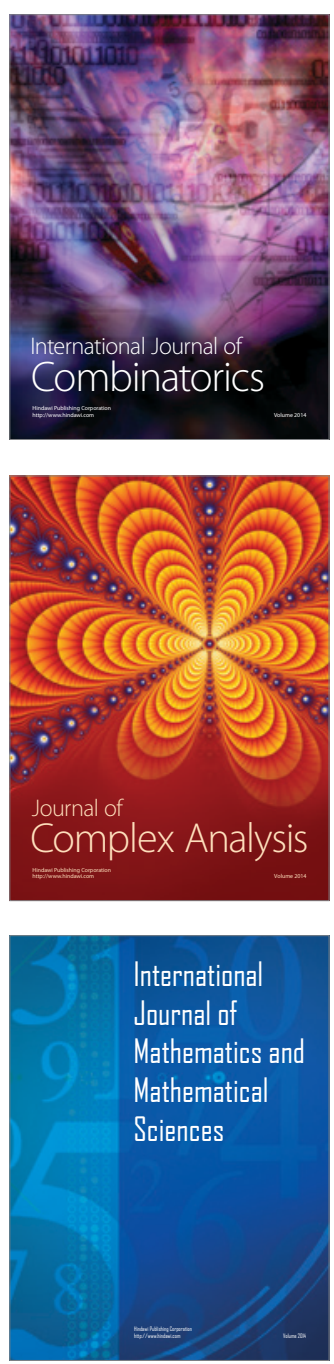
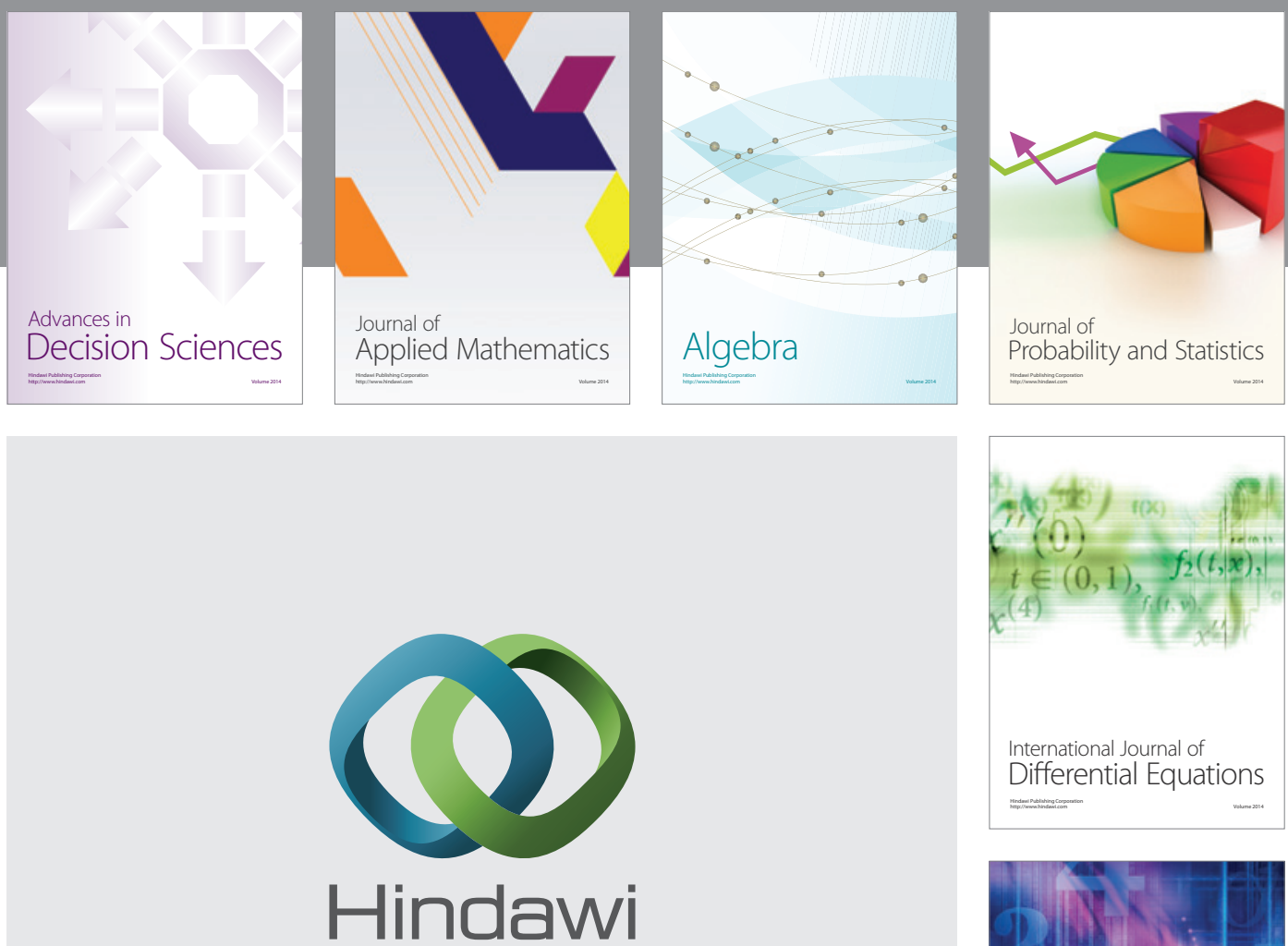

Submit your manuscripts at http://www.hindawi.com
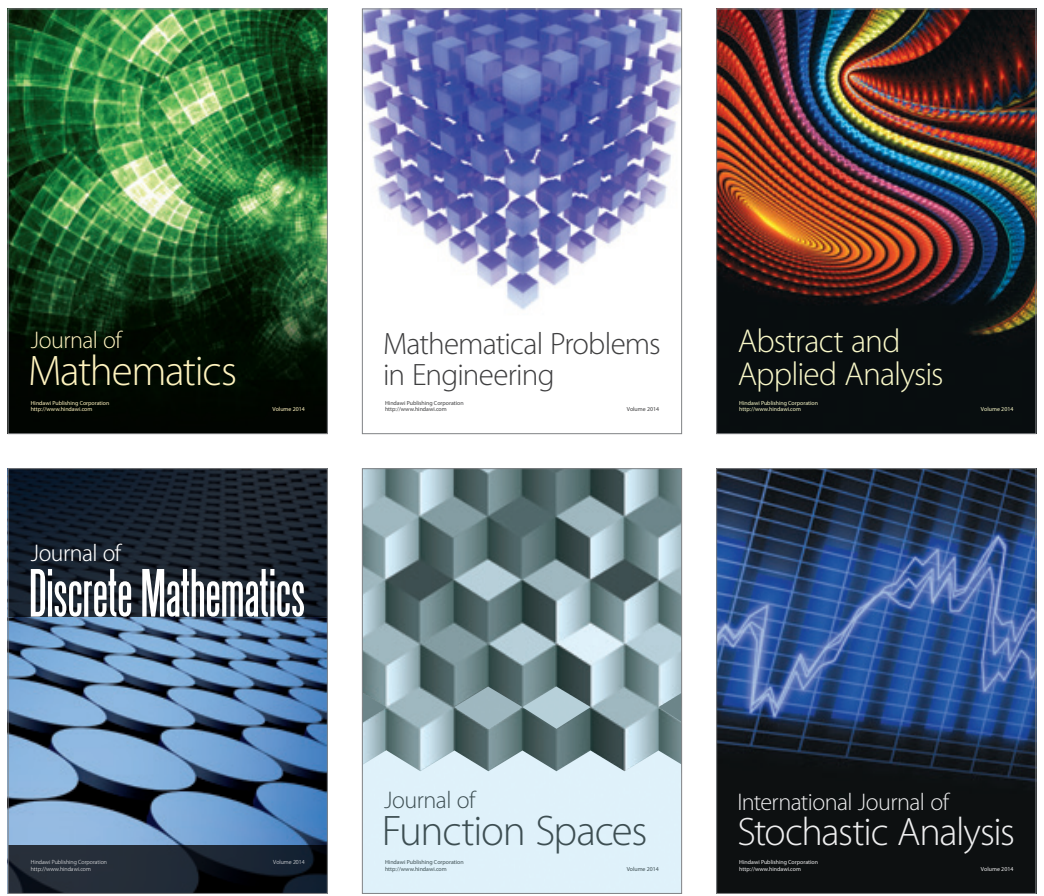

Journal of

Function Spaces

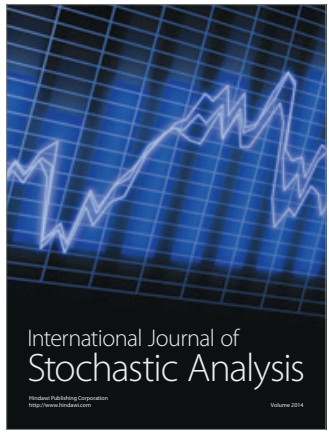

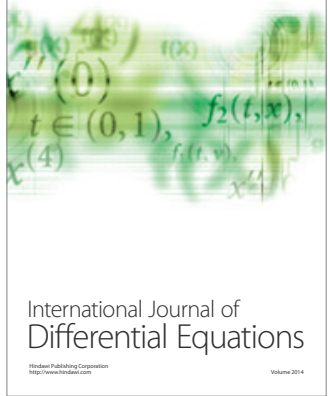
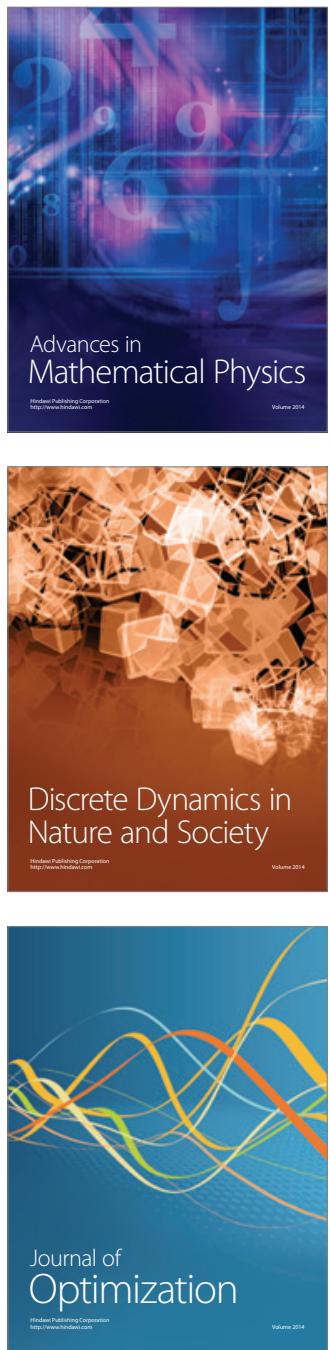